\title{
A Case of Proton Pump Inhibitor-induced Fasciitis
}

\author{
Yuki Nakanishi ${ }^{1}$, Shinichi Matsumoto ${ }^{2}$, Iori Motohashi ${ }^{2}$ and Toru Morikawa ${ }^{2}$
}

Key words: fasciitis, proton pump inhibitor, drug interaction

(Intern Med 58: 3203-3204, 2019)

(DOI: 10.2169/internalmedicine.2369-18)

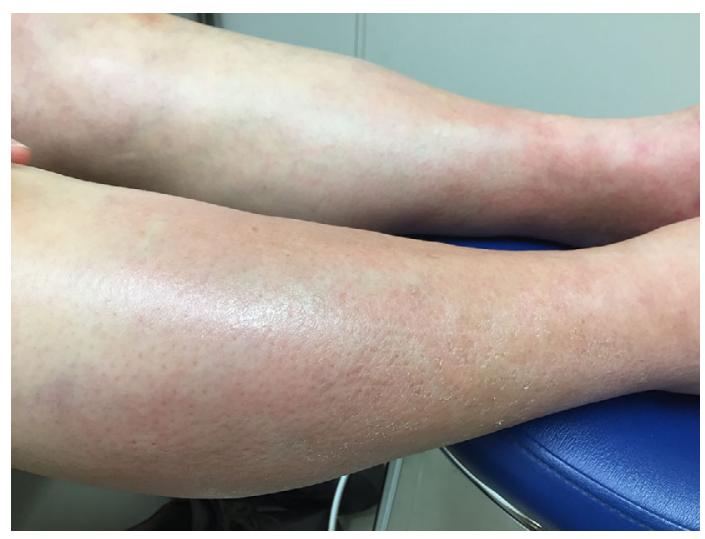

Picture 1.

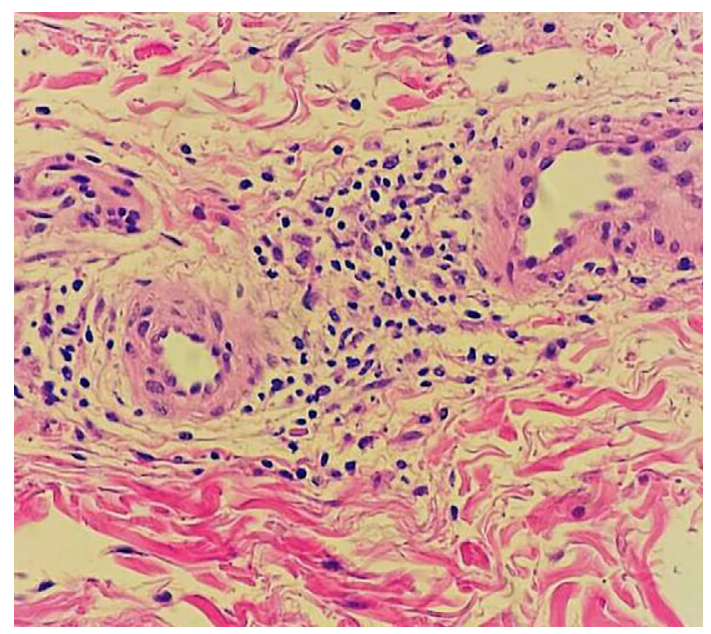

Picture 3.

A 70-year-old woman presented with symmetric swelling and pain in the lower legs that developed three weeks after she had started taking a proton pump inhibitor (PPI) for gastroesophageal reflux disease (GERD) (Picture 1). A physical examination did not reveal "peau d'orange" or "groove sign". The eosinophil count, and C-reactive protein, creatine

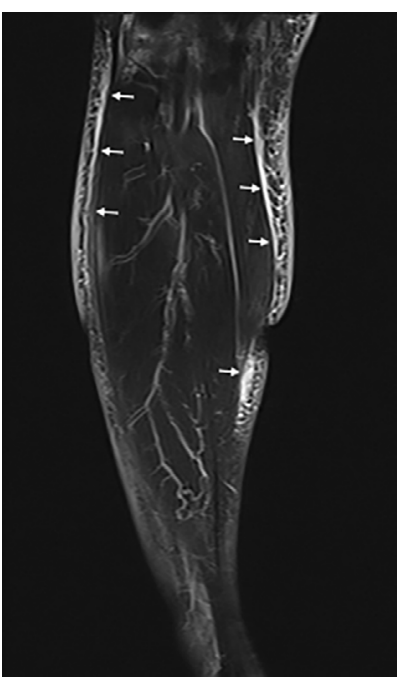

Picture 2.

kinase, aldolase, and soluble IL-2 receptor levels were within the normal ranges. Leg MRI revealed subcutaneous edema along the fascia (Picture 2). A skin punch biopsy specimen from the calf, which included the epidermis and dermis to subcutaneous fat, without the fascia, showed nonspecific lymphocyte infiltration in the dermis, but it did not reveal eosinophil infiltration or lymphoma cell infiltration within the small blood vessels (Picture 3). Thus, to rule out a drug interaction, we switched from PPI to histamine 2receptor antagonists, which alleviated her symptoms. Subsequently, her GERD symptoms worsened and the administration of the PPI was resumed. However, the swelling and pain were exacerbated again. Her symptoms resolved again with the discontinuation of the PPI, and a clinical diagnosis of PPI-induced fasciitis was suggested. Drug interactions are not necessarily associated with systemic involvement, such as drug-induced peripheral edema. Even in cases of localized involvement, it is important to differentiate a drug interaction. Eosinophilic fasciitis (EF) has been reported to occur without eosinophilia or eosinophilic infiltration in the fascia

${ }^{1}$ Department of General Medicine, Nabari City Hospital, Japan and ${ }^{2}$ Department of General Internal Medicine, Tokyo Joto Hospital, Japan Received: November 17, 2018; Accepted: April 24, 2019; Advance Publication by J-STAGE: July 10, 2019

Correspondence to Dr. Yuki Nakanishi, y_nakanishi_127@yahoo.co.jp 
and to be triggered by drugs (1). Thus, drug-induced EF without eosinophilia or eosinophil infiltration were possible diagnoses in the present case. However, the symptoms resolved after the discontinuation of the PPI and were exacerbated after its resumption, which suggests that the PPI provoked localized fasciitis. Although various adverse effects of PPIs have been reported (2), to our knowledge, this is the first report to describe PPI-induced fasciitis.

The authors state that they have no Conflict of Interest (COI).

\section{References}

1. Long H, Zhang G, Wang L, Lu Q. Eosinophilic skin diseases: a comprehensive review. Clin Rev Allergy Immunol 50: 189-213, 2016.

2. Yadlapati R, Kahrilas PJ. The "dangers" of chronic proton pump inhibitor use. J Allergy Clin Immunol 141: 79-81, 2018.

The Internal Medicine is an Open Access journal distributed under the Creative Commons Attribution-NonCommercial-NoDerivatives 4.0 International License. To view the details of this license, please visit (https://creativecommons.org/licenses/ by-nc-nd/4.0/).

(C) 2019 The Japanese Society of Internal Medicine Intern Med 58: 3203-3204, 2019 